\section{Intersections}

Canadian Journal of Music

Revue canadienne de musique
Intersections CANADIAN JOURAL OF MUSIC
REVUE CANADIENEE DE MUSIQUH

\title{
Lévi-Strauss, brèves réflexions sur ses inspirations, sa démarche et son legs
}

\section{Nathalie Fernando}

Volume 30, numéro 1, 2010

URI : https://id.erudit.org/iderudit/1003496ar

DOI : https://doi.org/10.7202/1003496ar

Aller au sommaire du numéro

\section{Éditeur(s)}

Canadian University Music Society / Société de musique des universités canadiennes

\section{ISSN}

1911-0146 (imprimé)

1918-512X (numérique)

Découvrir la revue

Citer cet article

Fernando, N. (2010). Lévi-Strauss, brèves réflexions sur ses inspirations, sa démarche et son legs. Intersections, 30(1), 5-21.

https://doi.org/10.7202/1003496ar

\section{Résumé de l'article}

Le présent article est issu d'une communication orale donnée à l'occasion d'une journée d'étude fêtant le centenaire de Lévi-Strauss. Cette dernière avait pour objectif de synthétiser brièvement la nature de son regard structural et les préoccupations d'ordre anthropologique qui sous-tendent sa réflexion. Elle tentait aussi de retenir les points forts de la recherche lévi-straussienne qui permettent encore aujourd'hui à nombre de sciences humaines de poser de nouveaux enjeux et de les traiter en retenant les leçons de l'histoire.

Face à une oeuvre colossale qui a maintes fois été analysée, ma démarche consiste à m'intéresser davantage à ce que Lévi-Strauss révèle de son parcours intellectuel dans son oeuvre, mais aussi au cours des multiples entretiens qu'il a donnés - notamment ses sources d'inspiration, ce qu'il dit de ce qu'il a écrit, et la façon dont cela a été reçu, à savoir les critiques qui lui ont été faites mais aussi les réponses qu'il a apportées.

Il s'agit en quelque sorte d'envisager l'homme dans sa démarche d'anthropologue, mais aussi d'évoquer Lévi-Strauss vu par Lévi-Strauss au coeur des sciences humaines, milieu propice — et c'est naturel — aux débats effervescents.
All Rights Reserved (C Canadian University Music Society / Société de musique des universités canadiennes, 2011
Ce document est protégé par la loi sur le droit d'auteur. L’utilisation des services d'Érudit (y compris la reproduction) est assujettie à sa politique d'utilisation que vous pouvez consulter en ligne.

https://apropos.erudit.org/fr/usagers/politique-dutilisation/ 


\title{
LÉVI-STRAUSS, BRÈVES RÉFLEXIONS SUR SES INSPIRATIONS, SA DÉMARCHE ET SON LEGS ${ }^{1}$
}

\author{
Nathalie Fernando
}

\begin{abstract}
Dans le présent texte, nous reprenons les paroles introductives d'une journée d'étude organisée en l'honneur des cent ans de Lévi-Strauss. Entre cette journée festive et ces quelques pages, Lévi-Strauss nous a quittés. Que le lecteur trouve ici un modeste témoignage de reconnaissance envers l'un des plus grands anthropologues du $\mathrm{XX}^{\mathrm{e}}$ siècle, non seulement pour son œuvre, mais encore pour les perspectives qu'il a su tracer, notamment à tous ceux qui sont curieux de mieux comprendre l'unité de l'homme au-delà de la diversité des cultures. Car comme le soulignaient justement Marc Kirch et Patricia Legou dans l'éditorial de La lettre du Collège de France publiée à cette même occasion, "Peu d'hommes ont autant de titre à prononcer le vers magnifique et redoutable de Terence : Homo sum, et humani nihil a me alienum puto. Je suis homme, et rien de ce qui est humain ne m'est étranger ${ }^{2}$
\end{abstract}

À travers son œuvre, Lévi-Strauss a tenté d'analyser et de théoriser l'univers symbolique humain. À l'heure d'aujourd'hui, nombre de problématiques qu'il a soulevées demeurent encore au cœur des discussions anthropologiques. L'objectif de cet article est de confronter la méthode structurale à ce qui a été dit à son égard, et à ce que Lévi-Strauss lui-même a révélé de ses inspirations théoriques et méthodologiques. Par ce biais se dessinent ses convictions scientifiques et philosophiques, nées à n'en pas douter de son profond respect pour l'Homme, mais surtout pour la vie qui jaillit à travers lui et qui échappe à sa propre condition.

Tout en étant consciente que notre propos représente en cela une esquisse bien modeste et non sans défaut d'un entreprise immense, nous aurons ainsi tenté de poser un regard ethnologique sur l'œuvre de ce chercheur, à la façon dont il aurait peut-être apprécié qu'on le fît, c'est-à-dire sous la forme d'une "psychologie», tout en nous remémorant ses paroles :

Ce que nous allons chercher à des milliers de kilomètres ou tout près, ce sont des moyens supplémentaires de comprendre comment fonctionne l'esprit humain. Nous faisons donc un genre de psychologie. Et ce qui est vrai pour des objets l'est encore plus quand on considère les croyances, les coutumes, les institutions (1988, p. 153).

1 Je remercie sincèrement Nicole Revel pour ses relectures attentives et ses précieux conseils.

2 La Lettre du Collège de France. Claude Lévi-Strauss, centième anniversaire. Hors série, Novembre 2008 . 
Il va sans dire que les lignes qui suivent doivent beaucoup aux spécialistes de l'œuvre de Lévi-Strauss 3 et à ceux qui ont minutieusement reporté les entretiens qu'il leur a accordés. Qu'ils soient ici remerciés pour la lumière qu'ils ont su apporter à la lecture d'une œuvre dense et complexe, dont nous n'avons pu apprécier l'apport qu'a posteriori, dans le domaine qui est le nôtre, celui de l'ethnomusicologie.

\section{UNE INTUITION ET DES RENCONTRES À L'ORIGINE D'UNE VOCATION}

Lévi-Strauss a dit : «je suis un Kantien vulgaire» et «peut-être structuraliste de naissance». De Kant, il a retenu «que l'esprit a ses contraintes, qu'il les impose à un réel à jamais impénétrable, et qu'il ne le saisit qu'à travers elles» (1988, p. 152). Quant au «structuralisme», l'anthropologue en a fait une grille d'analyse fondée sur la notion d'opposition, dans la perspective d'une approche comparative. Les objets sont alors analysés sous l'angle de leurs écarts différentiels, et les relations d'inversion et de réciprocité qu'ils entretiennent à divers niveaux sont traduites en un modèle opératoire abstrait. Ce dernier prend sens au sein d'une structure qui rend compte des logiques sous-jacentes et inconscientes, susceptibles de symboliser le lien intangible, mais inéluctable, entre le sensible et l'intelligible.

La méthode structurale aura cherché :

à transcender l'opposition du sensible et de l'intelligible en [se] plaçant d'emblée au niveau des signes. En effet, ceux-ci expriment l'un par le moyen de l'autre. Même en très petit nombre, ils se prêtent à des combinaisons rigoureusement agencées qui peuvent traduire, jusqu'en ses moindres nuances, toute la diversité de l'expérience sensible. Ainsi espéronsnous atteindre un plan où les propriétés logiques se manifesteront comme attributs des choses aussi directement que les saveurs, ou les parfums dont la particularité excluant toute méprise renvoie cependant à une combinaison d'éléments qui, diversement choisis ou disposés, eussent suscité la conscience d'un autre parfum. Grâce à la notion de signe, il s'agit donc pour nous, sur le plan de l'intelligible et non plus seulement du sensible, de rendre des qualités secondes au commerce de la vérité (1964, p. 22).

Dans l'œuvre de Lévi-Strauss, la mise au jour de la dialectique nécessaire, voire de l'interpénétration de paradigmes longtemps opposés, en anthropologie comme en philosophie, tels ceux du sensible et de l'intelligible, ou encore de la nature et de la culture font l'objet, même aujourd'hui, de bien des débats. La nature étant la source pour l'homme "de l'émotion esthétique et des premières spéculations» (1983, p. 166), une inspiration constante au sein de laquelle et en interaction avec laquelle il construit son identité et sa spécificité. En ayant explicitement réintroduit le débat à cet égard, il démontre la nécessité pour les sciences humaines de s'extirper un jour définitivement de l'opposition qu'elle a tendance à conserver entre le biologique et le culturel en concédant ça et là des passerelles 
éphémères entre les deux, permettant d'expliquer la place, le rôle et le statut de l'homme au milieu d'un univers qu'il aurait à dompter, ou auquel il serait soumis. Lévi-Strauss prédit ainsi que "si la distinction entre nature et culture venait un jour à s'estomper, le rapprochement ne se produirait pas à ce qu'on appellerait, dans le langage d'aujourd'hui l'interface des phénomènes humains et animaux", mais «entre ce qu'il y a de plus élémentaire, de plus fondamental dans les mécanismes de la vie, et ce qu'il a de plus complexe dans les phénomènes humains» (1983, p. 149). Ainsi, il replace la problématique au niveau de la définition du symbolique et de la comparaison de toute forme de vie.

La méthode structurale a constitué en premier lieu une manière de traiter de la relation entre le réel et le symbolique. Elle a ainsi eu pour ambition de formaliser au niveau le plus abstrait des principes inconscients et inhérents à la pensée humaine, mais détenant une valeur collective et transculturelle.

À travers la grille structurale, guidé par les tendances naturelles de son esprit et poussé par le désir de faire émerger le sens d'un chaos apparent bien que nécessairement ordonné, Lévi-Strauss a vu et analysé le monde. La révélation - suscitée par l'observation de la nature - aurait eu lieu, dit-il, lorsque, en mission sur la ligne Maginot et étendu dans l'herbe, il se serait interrogé sur les mystères de la composition admirable d'une fleur, laquelle ne devrait rien au hasard (Beuchot 2006). Immédiatement, il s'interroge sur l'éventualité que d'autres pans de la nature, y compris humaine, se nourrissent, se construisent et se correspondent sur la base de structures sous-jacentes répondant à des logiques similaires. Il verra dans la linguistique et les méthodes qu'elle a développées le moyen de répondre à ses interrogations, qu'il aborde, aussi, à travers d'autres disciplines.

Philosophe de formation, Lévi-Strauss a le goût des idées, des débats politiques, mais affiche aussi ostensiblement sa passion pour la peinture, la musique et l'art en général. Il n'entend pas cloisonner ses connaissances et ses compétences de même que celles de l'Homme qu'il observe et qu'il cherche à définir : il privilégiera toujours les relations entre les objets et les phénomènes, les comparaisons entre les cultures et leurs savoir-faire. Il insiste sur le fait que

l'une des différences essentielles entre la manière dont nous réfléchissons et la manière dont ces peuples réfléchissent, c'est notre besoin de morceler. Nous avons appris cela de Descartes : diviser la difficulté en autant de parcelles qu'il est requis pour la mieux résoudre. La pensée des peuples dits primitifs récuse ce morcellement. Une explication ne vaut qu'à condition d'être totale. Lorsque nous cherchons la solution d'un problème particulier, nous nous adressons à telle ou telle discipline scientifique ou bien au droit, à la morale, à la religion, à l'art [...] Pour les peuples qu'étudient les ethnologues, tous ces domaines sont liés. Aussi chaque expression de la vie collective constitue ce que Mauss appelait un fait social total. Elle met simultanément en cause tous ces aspects». (1988, p. 157-158).

Mais les différences qu'il note sont à mettre sur le compte de la diversité des traditions et des savoir-faire. Car fondamentalement, sa théorisation du symbolique ne peut tenir sans l'hypothèse de l'existence de principes et de 
capacités opératoires qu'il considère comme les fondements inconscients, universels et propres au fonctionnement de l'esprit humain en général.

Dans Tristes tropiques, Lévi-Strauss avoue avoir une «intelligence néolithique» et «se déplace(r) sur une frontière toujours mouvante» (1988, p. 133), sans nécessairement faire fructifier les connaissances acquises. Toutefois, on devine à travers son œuvre une pensée transversale, constituée - telle sa méthode - de strates entrecroisées. Sa réflexion, sans cesse évolutive, peut mener à ce que l'on perçoit comme des contradictions ou des paradoxes. Mais LéviStrauss est surtout doté d'une sensibilité intuitive lui permettant de saisir les phénomènes de façon empirique, avant d'en avoir véritablement saisi les ressorts et avant même que son esprit et sa méthode ne les rationalisent en les décomposant, pour mieux les recomposer à un niveau plus abstrait.

Sans aucun doute, Lévi-Strauss doit son inspiration méthodologique à sa curiosité sans limite ni frontière, mais aussi à son parcours atypique, soumis aux aléas de l'histoire. Ayant dû fuir le nazisme, il se réfugie aux États-Unis où son esprit cartésien entre en résonance avec la rigueur de l'analyse linguistique. De fait, il devient le témoin des multiples débats qui animent l'anthropologie américaine au milieu du $\mathrm{XX}^{\mathrm{e}}$ siècle.

Dans les inspirations qu'on lui reconnaît et auxquelles il fait volontiers référence dans nombre d'entretiens - mais dont il dit aussi se distancier sur certains points -, on retrouve des figures de proue de l'ethnologie, de l'anthropologie, de la linguistique, de la psychanalyse, de la socio-politique. C'est dire son intérêt pour toutes les formes de savoir susceptibles de fournir les méthodes nécessaires à la résolution des problématiques qui le préoccupent.

Il évoque ainsi la mémoire de Frantz Boas, qui a scientifiquement su démontrer l'absurdité du racisme en ayant mis en avant le fait que l'unité d'une société relève non pas de l'identité biologique, mais de la culture. Il retient également, des propos de l'anthropologue, que les lois du langage fonctionnent dans l'inconscience du sujet et qu'à ce titre, il est dès lors possible de les étudier comme des objets. La pensée de Lévi-Strauss n'est pas non plus hostile à «l'ordre des possibles» dans lequel l'homme piocherait et dont parlait Ruth Benedict. À ce titre, il conçoit le primat de l'infrastructure comme un jeu de cartes qui constituerait une

«donnée de l'histoire et de la civilisation», dont la distribution se fait à «l'insu» des joueurs, «avec des données subies, mais que chaque société, comme chaque joueur, interprète dans les termes de plusieurs systèmes, qui peuvent être communs ou particuliers : règles d'un jeu, ou règles d'une tactique. Et l'on sait bien qu'avec la même donne des joueurs différents ne fourniront pas la même partie, bien qu'ils ne puissent, contraints aussi par des règles, avec une donne quelconque, fournir n'importe quelle partie (1962b repris in 2008, p. 658).

Quant à la méthode comparative dont il a généralisé le principe au travers de l'analyse structurale - et pour l'application de laquelle il recommande de s'assurer au préalable d'une connaissance ethnographie approfondie et équitable entres les cultures impliquées dans la comparaison (histoire commune, aire 
géographique délimitée, etc.) - il n'exclut pas l'éventualité d'y soumettre des objets ou des principes qui ont traversé le temps et seraient issus de traditions tout à fait différentes.

L'idée que l'homme bénéficie, au-delà de certaines contraintes relatives à son milieu, son histoire et ses capacités, d'une liberté de choix, sur la base de critères qu'aujourd'hui l'on nommerait volontiers «esthétiques", entre alors de fait dans les paramètres qui contribuent à la dynamique des cultures. À cela s'ajoutent les phénomènes d'emprunt puis de réappropriation selon les catégories linguistiques propres à chacune des sociétés, lesquelles ne peuvent être envisagées comme fermées sur elles-mêmes.

Il retiendra tout particulièrement la pensée de Marcel Mauss et sa théorie $\mathrm{du}$ «fait social total». Pour Lévi-Strauss, l'Essai sur le don a été une véritable révélation dont il s'est inspiré pour l'étude du système de parenté. Le don interrelie divers pans de la société et sous-tend des normes collectivement reconnues. Dans cette perspective, la méthode structurale a formulé l'analyse des échanges dans ses aspects les plus profonds, leur présupposant un modèle de fonctionnement inconscient :

Pour comprendre convenablement un fait social, il faut l'appréhender totalement, c'est-à-dire comme une chose, mais comme une chose dont fait partie intégrante l'appréhension subjective (consciente et inconsciente) que nous en prendrions si, inéluctablement hommes, nous vivions le fait comme indigène au lieu de l'observer comme ethnographe. (1950, p. XXVIII).

Sa démarche semble fondée sur un fort désir de rendre compte du réel pensé par une explication rationnelle, sans pour autant sacrifier à une certaine subjectivité interprétative tout aussi inhérente à l'homme que le pouvoir de raisonner. Toutefois, il semble que les démarches de Mauss et de Lévi-Strauss soient inverses en ce que le premier envisage le don comme un geste symbolique qui traduit une obligation sociale et le second tente d'expliquer cette pratique sociale au regard de processus symboliques, accordant à ces derniers des dimensions opératoires au-delà de la structure qu'ils représentent.

Pendant sa jeunesse, Lévi-Strauss lit Freud et se trouve alors fasciné non pas tant par ses théories que par l'idée qu'une partie du sens relève de l'inconscient. L'analyse vise alors à rendre conscient ce qui ne l'est pas nécessairement - ce à quoi il s'emploiera dans l'étude des mythes. Freud démontre en effet que l'on peut comprendre de façon rationnelle des choses qui semblent ne pas l'être. Toutefois, Lévi-Strauss considérera comme un échec le fait que la psychanalyse se complaise dans un discours analytique portant sur un symbolique qui n'est plus fermement attelé au concret. Sceptique sur la recherche de l'explication causale dans le passé de l'individu et dans les pulsions biologiques, LéviStrauss s'est employé «à concevoir l'activité inconsciente de l'esprit comme une activité qui consiste non pas tant en des souvenirs antérieurs, ou postérieurs à la naissance, qu'en des règles de fonctionnement» (2000, p. 17).

Son engagement politique le conduit à lire Marx, dont il retient surtout l'usage de la notion de modèle et une réflexion qui ne se détache pas de l'analyse 
des conditions pratiques de l'existence. Lévi-Strauss avoue lui être resté philosophiquement attaché, et compare la démarche de ce dernier à celle de Freud en ce que l'objectif était d'expliciter la logique des comportements, au-delà de l'apparence. Mais pour Lévi-Strauss, ce ne sont pas de simples rapports de réflexion qui prévalent entre l'infrastructure et la superstructure : la relation entre les deux implique tout un ensemble d'inversions et de transformations très complexes qui "appartiennent toutes à un même groupe» et qui passent par un «opérateur » qu'il assimile à un

schème conceptuel qui s'intercale entre praxis et pratique et par l'opération duquel une matière et une forme dépourvues l'une et l'autre d'existence indépendante s'accomplissent comme structure, c'est-à-dire comme être à la fois empirique et intelligible. C'est à cette théorie des superstructures, à peine esquissées par Marx, que nous souhaitons contribuer, réservant à l'histoire - assistée par la démographie, la technologie, la géographie historique et l'ethnographie - le soin de développer l'étude des infrastructures proprement dites, qui ne peut être principalement la nôtre, parce que l'ethnologie est d'abord une psychologie (1962b, repris in 2008, p. 696).

L'inspiration majeure, celle qui a ébranlé toutes les autres et leur a sans doute donné sens a posteriori, est venue de sa rencontre avec Roman Jakobson grâce à qui il découvre les principes de la linguistique structurale. Pourquoi a-t-il été attiré par cette approche?

Elle n’a pas seulement renouvelé les perspectives linguistiques : une transformation de cette ampleur n'est pas limitée à une discipline particulière. La phonologie ne peut manquer de jouer, vis-à-vis des sciences sociales, le même rôle rénovateur que la physique nucléaire, par exemple, a joué pour l'ensemble des sciences exactes. [...] quand un évènement de cette importance prend place dans l'une des sciences de l'homme, il est non seulement permis aux représentants des disciplines voisines, mais requis d'eux, de vérifier immédiatement ses conséquences, et son application possible à des faits d'un autre ordre (1958, p. 39-40).

On reconnaîtra alors que l'un des apports ultérieurs de Lévi-Strauss, qui est au cœur des sciences humaines d'aujourd'hui, aura en effet été d'introduire implicitement la notion d'interdisciplinarité et, à travers elle, l'idée de la nécessaire fécondation réciproque de paradigmes analytiques développés dans des champs différents. Il apparaît comme une évidence, au fil de ses rencontres et ensuite de son œuvre, que Lévi-Strauss cherche à comprendre le mode de fonctionnement de l'individu considéré dans la totalité de son être, de façon à éviter de se perdre dans une «multitude d'explications particulières». Dans cette perspective, on peut émettre l'hypothèse que ses tentatives homologiques sont motivées par la conviction qu'il existe des processus symboliques similaires au service de savoir-faire divers et non par la similitude formelle de leurs manifestations. La variété des productions symboliques émaneraient d'un mode de fonctionnement dont les principes seraient assimilables à un petit nombre de règles, souples, adaptables et à géométrie variable selon les cultures. 


\section{BREF RETOUR SUR LES PRINCIPAUX AXES}

\section{DE LA PENSÉE STRUCTURALE}

Pour Lévi-Strauss, la méthode structurale visait à introduire de la clarté dans une discipline, l'anthropologie où, selon lui, régnait beaucoup de confusion. Il souhaitait rendre compte du fonctionnement de l'univers symbolique par l'élaboration d'un modèle simple pouvant s'appliquer à plusieurs domaines de la culture (structure de la société, des rituels, des mythes, des institutions, du langage, des relations entre la nature et la culture, de la musique, des arts plastiques, etc.). La structure relève du non observable. Elle est reconstruite par le chercheur. Elle renvoie à l'analyse de la relation entre les éléments, puis à la déduction d'un modèle de fonctionnement qui révèle des logiques inconscientes. Rappelons cependant que, hors du champ de la linguistique, la notion de «structure» n'a pas fait l'objet d'un consensus parmi les ethnologues. La structure, vue par Lévi-Strauss, détient un caractère systémique qui implique une relation réciproque entre les éléments, telle qu'une modification de l'un d'eux entraîne une modification des autres. La nature des relations entre les éléments est oppositive - on a souvent parlé de la recherche de "paire d'oppositions » - et le modèle intègre chaque transformation comme lui appartenant en propre. Ce dernier est construit de façon à rendre compte de la réalité observable et de toutes ses variations qui sont alors prévisibles, bien que toutes les solutions possibles ne seront pas nécessairement choisies par les individus. Si le modèle n'est pas nécessairement appliqué comme tel, il constitue toutefois une norme à laquelle la société se réfère. Dans l'analyse des mythes, Lévi-Strauss justifie l'intérêt de la mise au jour du modèle par le fait que les individus percevraient chacun un contenu différent pouvant toutefois se résumer à travers un petit nombre de traits invariants. Scubla souligne, en se référant aux cours que Lévi-Strauss donnait à l'École pratique des hautes études au sujet de l'Étude comparée des peuples sans écriture, que

l'étude des mythes ne consiste pas à retrouver un récit singulier et prototypique dont les diverses variantes constitueraient des altérations; mais elle a pour objectif de reconstituer l'ensemble des variantes ou «transformations» possibles d'un récit, qui ne devrait lui-même son statut de mythe qu'au fait d'appartenir à un tel «groupe de transformations» (1998, p. 13).

L'introduction de la notion de transformation est de nouveau issue d'une autre discipline - la lecture d'un ouvrage du biologiste D'Arcy Wentworth Thompson, On Growth and Form (1917), l'ayant alors inspirée. Ce système de transformation se retrouve dans l'espace-temps et d'une société à l'autre. Il s'agit, pour Lévi-Strauss, de définir le fait que des oppositions logiques perdurent au sein de la structure de tel ou tel mythe que l'on viendrait à comparer. La notion de transformation ne vise qu'à rendre compte de la diversité des variantes qui s'exprime à travers les mythes, à condition de bien identifier les principes d'inversion qui s'opèrent. C'est ce qui lui aura été reproché comme une forme d'idéalisation de la structure. 
L'analyse du mythe et le modèle que l'on en dégage sont élaborés comme des outils permettant de comprendre l'articulation des autres pans de la société et d'en révéler le sens. Les mythes ne sont pas analysés pour la signification de leur contenu mais parce qu'ils signifient au-delà de la conscience qu'en ont ceux qui les racontent. Cela autorise une analyse qui fait abstraction du sujet, d'où la phrase énigmatique : «les mythes se pensent entre eux» (1964, p. 20).

Ainsi, les éléments de la pensée structurale se trouvent réunis : la notion d'infrastructure inconsciente, sa manifestation consciente, la mise au jour du sens en déplaçant l'attention des termes aux relations qu'ils entretiennent, le tout formant un système fondé sur des lois générales qui tiennent compte de «l'arbitraire du signe» selon les cultures - c'est-à-dire de la signification qui lui est attribuée au sein de chaque société. Enfin, la valeur universelle de la structure se réclame de l'universalité des caractères humains à travers le temps et l'espace. Henri Chiarrucci le souligne :

Le structuralisme s'intéresse non pas à l'objet en soit [...] mais uniquement au système relationnel de l'objet. C'est ainsi qu'il est possible de trouver les mêmes structures dans des domaines différents, d'abolir les frontières entre les disciplines, et de reconnaître à la base de tout système, de toute pensée, un nombre limité de structures s'appliquant à des objets qui, eux, sont différents.

Dès lors, on doit admettre que lorsqu'on constate l'identité de principes structuraux, on découvre, en dernier ressort, une propriété de l'homme; l'étude des systèmes concrets n'aura servi qu'à élucider ses structures mentales ou mieux, pour citer Lévi-Strauss, "Les modalités temporelles des lois universelles en quoi consiste l'activité inconsciente de l'esprit». C'est ici que la praxis rejoint la Critique de la raison pure. Il n'est pas étonnant que l'on retrouve les mêmes propriétés structurelles dans le tableau de Mendelejev et dans la molécule d'ADN, siège des mécanismes héréditaires. L'un et l'autre ne sont, en effet, que la projection de nos structures mentales, qui interprètent des réalités dont l'essence, le noumène, reste par ailleurs totalement inconnaissable. Dans une telle perspective, chercher une explication de (par exemple) la musique revient à étudier non seulement les structures perceptives humaines, mais aussi certains patterns spécifiques, établis par le milieu culturel. La musique, en effet, trouve ses racines, ses fondements, dans des réalités aussi bien naturelles que culturelles [...]. (1973, p. 13)

L'analyse structurale permet d'expliquer ainsi les représentations totémiques : "Ce ne sont pas les ressemblances mais les différences, qui se ressemblent» (1962b, p. 115) Dans le domaine de recherche qui est le nôtre - celui de la catégorisation des patrimoines musicaux - c'est un système d'opposition qui apparaît après une analyse contrastive de la matière musicale d'une part, et du contexte dans lequel elle s'inscrit d'autre part, qui permet d'expliquer le lien entre ces deux pans de la culture. L'équipe de Simha Arom (Arom et al. 2009) a bien montré comment l'on pouvait, au sein des patrimoines musicaux de tradition orale, mettre en évidence la logique des représentations vernaculaires grâce à l'analyse du système de renvoi qui prévaut entre les trois domaines 
du symbolique que sont le langage, les croyances et la musique. Comment pourrait-on dégager une modélisation de ces principes? Il s'avère en effet que

chaque communauté ordonne les pièces qui composent son patrimoine musical en un certain nombre d'ensembles. La pertinence culturelle de ces ensembles est attestée par un nom spécifique et par le fait que chacun est lié à une ou plusieurs circonstances et/ou fonctions précises. Il en découle que l'organisation vernaculaire de ce patrimoine obéit nécessairement à une articulation cohérente. [...] le recoupement des données concernant les circonstances d'exécution avec les ensembles de pièces qui leur sont attachés et leurs dénominations respectives dans la langue autochtone fait apparaître la prépondérance des paramètres musicaux quant à la discrimination des catégories vernaculaires qui forment le patrimoine musical d'une communauté. Ces paramètres concernent tant la nature des formations instrumentales et vocales que les caractéristiques de la substance musicale» (Arom et al. 2009, p. 274).

Les écarts différentiels qui distinguent les ensembles de pièces appartenant à une communauté concordent avec une distinction faite par les détenteurs de la tradition lors des circonstances auxquels sont culturellement attachés ces ensembles. Un jeu d'écho des différences se dessine en inscrivant les divers pans de la culture dans un système régi par des principes similaires. Ces derniers opèrent au niveau du symbolique et peuvent demeurer implicites. Mais l'analyse sert, ainsi que l'a formulé Lévi-Strauss, à «définir une loi d'équivalence entre des contrastes significatifs qui se situent sur plusieurs plans» (1962b, repris in 2008, p. 654).

Quant à l'analogie entre mythe et musique, Lévi-Strauss la fonde sur le principe de «la double articulation» en linguistique. Il revient sur le fait que le mythe puise dans un stock de possibilités des éléments qui ne sont pas nécessairement compréhensibles sur le plan syntaxique parce qu'un ordre plus complexe les organise simultanément, celui des strates superposées.

Tout comme dans le langage parlé, les échelles musicales puisent dans tous les possibles du continuum sonore. La composition musicale se comprend à la fois harmoniquement et syntaxiquement, ainsi que par une accumulation de repères qui ne font sens que lors de l'exécution de l'œuvre. Les études les plus récentes portant sur les systèmes scalaires (Arom, Fernando, Marandola, 2007), s'inspirent directement de la phonologie puisqu'elles tentent de comprendre les modalités de sélection des hauteurs au sein d'un continuum sonore et les raisons de l'instabilité de ces sélections au niveau de la performance. Elles ont été menées dans des communautés de traditions orales d'Afrique centrale, au Cameroun, auprès des populations pygmées Bedzan et Ouldémé. On peut gager cependant que les modèles abstraits définis dans ces contextes respectifs sont pertinents pour d'autres régions de l'Afrique subsaharienne.

La problématique consistait en ce qu'une pièce n'est presque jamais jouée deux fois avec la même échelle, au delà du problème que pose le non respect de la hauteur absolue dont on connaît le caractère inopérant dans cette région. Les tenants de ces traditions n'ont pas de termes pour nommer les échelles musicales, ce sont des catégories implicites. Il nous était donc impossible de 
communiquer avec eux sur le matériau musical. Pour autant, il nous fallait nous focaliser sur certains de ses aspects parmi les plus précis et dont on pouvait supposer - au même titre que la langue - qu'ils étaient régis par des lois dont l'usage demeure inconscient aux musiciens. Aussi, nous avons mis au point une méthode expérimentale permettant non seulement de manipuler le matériau musical à travers les pièces du patrimoine autochtone, mais encore de conserver le timbre des voix ou des instruments. Le support informatique nous a aidés à concevoir des échelles hypothétiques et à les appliquer à des polyphonies complexes. Une fois les procédures mises au point, nous pouvions cerner pas à pas les modalités de fonctionnement du système scalaire en éliminant peu à peu les paramètres non pertinents, voire en créant des artefacts n’ayant jamais été produits par les musiciens afin de tester le modèle présupposé et de le valider. L'expérimentation se déroulait en interaction constante avec les musiciens sur la base d'induction et de déduction. Les musiciens nous guidaient dans la compréhension de leur système scalaire par leurs réactions et leurs commentaires.

Les données qui résultent de cette expérimentation montrent bien le jeu des relations entre les termes, ainsi que le nécessaire maintien d'un équilibre au sein d'une forme globale afin que la matérialisation de la structure que nous avions dégagée, puis analysée à un niveau plus abstrait, soit considérée comme pertinente par les musiciens eux-mêmes. Le modèle qui a été défini dans ce cadre manifeste une puissance dynamique en ce que le système de transformation à travers lequel il s'exprime repose sur la notion de contrainte réciproque. Par exemple, les échelles vocales bezan se fondent sur des intervalles structurant inférieurs à l'octave tels que des tricordes (intervalle comprenant lui-même deux intervalles conjoints) ou des tétracordes (intervalle comprenant trois intervalles conjoints). Chacun de ces intervalles-cadres peut faire l'objet de marges de réalisation relativement importantes, tout comme - dans une moindre mesure - les intervalles qui les composent. Mais ces marges de réalisation sont toutefois limitées par une triple contrainte, relative :

- aux limites inférieure et supérieure admises par les tricordes et tétracordes,

- aux limites inférieure et supérieure de l’octave, formées par la juxtaposition de ces intervalles-cadres et

- par les valeurs minimales que peuvent prendre les intervalles qui constituent les intervalles-cadre.

Il y a là un jeu relationnel entre les intervalles et lorsque les modifications que nous opérions sur le matériau musical ne respectaient pas les limites de ces règles, lesquelles ont pu être précisément définies en termes de mesures, les échelles étaient rejetées par les musiciens. On est alors en présence de ce que les linguistes désignent par «l'aire de connotation d'un phonème».

Si l'on s'interroge enfin sur la signification musicale de la musique sérielle et concrète et l'appréciation très critique que Lévi-Strauss en a formulée, on peut immédiatement supposer qu'il y a là un malentendu conceptuel et un hiatus entre la méthode et les faits. Il semble que les compositeurs aient utilisé la méthode structurale comme support à l'élaboration de processus compositionnels, 
alors que la démarche de Lévi-Strauss avait pour finalité de développer un modèle abstrait à partir de l'observation empirique de faits, et dont la portée était transculturelle. L'anthropologue et les compositeurs auraient en cela suivi des chemins opposés. Le nœud du problème réside, on peut le supposer, en ce que les stratégies cognitives qui conduisent à l'abstraction du modèle de Lévi-Strauss sont très différentes de celles qui, lors de l'écoute des œuvres, président à la perception de ce modèle devenu structures sonores. Par ailleurs, les méthodes de composition ne se construisent pas au niveau de l'inconscient des compositeurs et ne peuvent être appréciées que lorsque la série est révélée à l'auditeur qui doit la repérer et la garder en mémoire afin de donner un sens à l'œuvre sérielle. Or la structure dont parle Lévi-Strauss opère dans l'inconscient et à l'insu du sujet. L'analyse structurale ne prive pas le sujet d'une compréhension sensible de l'objet en dehors de toute conscience de sa structure : chaque mythe a un sens qui lui est propre et dont chacun des membres de la communauté est capable de rendre compte à sa manière. Mais «les mythes se pensent dans les hommes et à leur insu » (1964, p. 20). Malgré les différences qui prévaudront d'une interprétation à une autre, les caractéristiques du mythe se réduiront à un petit nombre de traits invariables. Dans le cadre des musiques sérielles et concrètes, le sens émane de la relation entre les notes et se trouve exclusivement relatif à la structure primordiale de l'œuvre.

La sensibilité et l'attrait de Lévi-Strauss pour les arts, de même que son attachement profond pour les diverses manifestations des capacités symboliques humaines, nous conduisent à penser que la correspondance entre les formes plastiques, la musique et le récit s'est imposée à lui comme une démarche nécessaire. Il semblait en effet imaginer ces trois pôles comme des medium privilégiés de significations qui, au-delà des mots, forment d'autres types de langages. Ceux-ci atteindraient les profondeurs de l'affectivité par l'expérience esthétique mais n'en seraient pas pour autant dénués de fondement logique que le modèle structural permettrait précisément de mettre en évidence. La correspondance entre mythe et musique lui inspire une pensée empreinte de lyrisme :

La vraie réponse se trouve, croyons-nous, dans le caractère commun du mythe et de l'œuvre musicale, d'être des langages qui transcendent, chacun à sa manière, le plan du langage articulé, tout en requérant comme lui, et à l'opposé de la peinture, une dimension temporelle pour se manifester. Mais cette relation au temps est d'une nature assez particulière : tout se passe comme si la musique et la mythologie n'avaient besoin du temps que pour lui infliger un démenti. L'une et l'autre sont, en effet, des machines à supprimer le temps. Au-dessous des sons et des rythmes, la musique opère sur un terrain brut, qui est le temps physiologique de l'auditeur; temps irrémédiablement diachronique puisqu'irréversible, et dont elle transmute pourtant le segment qui fit consacré à l'écouter en une totalité synchronique et close sur elle-même. L'audition de l'œuvre musicale, du fait de l'organisation interne de celle-ci, a donc immobilisé le temps qui passe; comme une nappe soulevée par le vent, elle l'a rattrapé et replié. Si bien qu'en écoutant la musique et pendant que nous l'écoutons, nous accédons à une sorte d'immortalité (1964, p. 24). 
Plus tard, dans un entretien qu'il accordera à Jean-Jacques Nattiez, il avouera sa passion pour la composition. Nattiez traduit ainsi les propos de l'anthropologue :

S'il analyse les mythes, c'est pour compenser un manque, le désir et la nostalgie de ne pas être compositeur. Les motifs personnels deviennent alors sources d'inspiration pour la recherche : la musique propose des formules et des schèmes pour interpréter des problèmes de mythologie (1973, p. 5).

Pour terminer, on ne peut passer sous silence la façon dont Lévi-Strauss tenait à distance les interprétations et la mode structurale auxquelles sa méthode avait pu donner lieu. Ainsi, il déclare, dans nombre d'entretiens, ne pas avoir strictement appliqué le modèle linguistique mais avoir entrevu en lui, le sens de ce qu'il cherchait ailleurs. Il se l'est en quelque sorte réapproprié, en a saisi l'essence en laissant son esprit intuitif trouver de nouveaux objets d'applications :

On a mal compris la nature et l'importance de mes emprunts à la linguistique. En dehors d'une inspiration générale [...] ils se réduisent au rôle de l'activité inconsciente de l'esprit dans la production de structures logiques [...]; ensuite, à ce principe fondamental, que les éléments constitutifs n'ont pas de signification intrinsèque; elle résulte de leur position. C'est vrai du langage et c'est aussi vrai pour d'autres faits sociaux. Je ne crois pas avoir demandé plus à la linguistique, et Jakobson, au cours de nos conversations, était le premier à reconnaître que, dans un autre domaine, je faisais un usage original de ces notions [...]. Lévi-Strauss ajoute : «en titrant "Anthropologie structurale», je voulais simplement dire que je me situais dans la même province intellectuelle que Saussure, Troubetzkoy, Jakobson, Benveniste ou tout au moins que c'était mon ambition (1983, p. 158).

\section{LES CRITIQUES ...}

On sait que les critiques n'ont pas manqué. Le principal reproche relève d'une sorte de paradoxe qui voudrait que cette méthode implique une dialectique permanente entre le concret et l'abstrait, alors que la structure serait fondée sur une spéculation du chercheur. La méthode structurale aurait également le défaut de vouloir se fonder, dans la théorie de l'échange, sur les principes d'équilibre et de réciprocité, en omettant d'intégrer les phénomènes d'instabilité et de conflits qui sont omniprésents dans la réalité.

On peut évoquer la controverse avec Edmond Leach sur la parenté et celle avec Sartre au sujet de Race et Histoire. La critique reposant sur la dissolution du sujet dans une analyse de nature exclusivement abstraite en est une autre. Or Lévi-Strauss, conscient de la différence entre les règles et le comportement des individus, différencie, lorsqu'il parle du système de parenté, le "système de dénomination" - la nomenclature - le «système de parenté» et le «système des attitudes» restituant au sujet sa liberté dans la contrainte de son action. Enfin, à l'effet de retrait systématique de la réalité pragmatique que l'analyse structurale induit, Barthes répond en sa faveur que «le grand apport 
méthodologique de Lévi-Strauss [...] c’est [...] d’avoir décroché les formes de leur contenu» (Barthes 1957, p. 235).

Selon une autre critique bien courante, la méthode structurale déshumanise la réalité et veut la réduire au système symbolique, mais aussi à un modèle mathématique issu d'une spéculation du chercheur. Envers cela, on ne saurait nier le goût de Lévi-Strauss pour la rationalisation formelle. Sa rencontre avec le mathématicien André Weil fut, en effet, capitale.

Dans la même veine, on reproche à Lévi-Strauss d'envisager le système hic et nunc en ne prenant pas en considération la diachronie. Son analyse formelle est fondée sur l'observation synchronique des faits et non sur la recherche historique des causalités. Car le regard que Lévi-Strauss porte sur l'histoire, telle qu'elle est racontée, un regard sceptique. Une telle conception de l'histoire relèverait presque du «mythe»:

L'ethnologue respecte l'histoire, mais ne lui accorde pas une valeur privilégiée. Il la conçoit comme une recherche complémentaire de la sienne : l'une déploie l'éventail des sociétés humaines dans le temps, l'autre dans l'espace. Et la différence est moins grande encore qu'il ne semble, puisque l'historien s'efforce de restituer l'image de sociétés disparues telles qu'elles furent dans des instants qui, pour elles, correspondirent au présent; tandis que l'ethnographe fait de son mieux pour reconstruire les étapes historiques qui ont précédé dans le temps les formes actuelles. [...] la diversité des formes sociales, que l'ethnologie saisit étalées dans l'espace, offre l'aspect d'un système discontinu; or on s'imagine que, grâce à la dimension temporelle, l'histoire nous restitue, non des états séparés, mais le passage d'un état à un autre sous une forme continue. Et comme nous croyons nous-mêmes appréhender notre devenir personnel comme un changement continu, il nous semble que la connaissance historique rejoint l'évidence du sens intime. [...] il n'est pas nécessaire de trancher le problème philosophique pour s'apercevoir que la conception qu'on nous propose de l'histoire ne correspond à aucune réalité. [...]. Chaque épisode d'une révolution ou d'une guerre se résout en une multitude de mouvements psychiques et individuels; chacun de ses mouvements traduit des évolutions inconscientes, et celles-ci se résolvent en phénomènes cérébraux, hormonaux, ou nerveux, dont les références sont elles-mêmes d'ordre physique ou chimique ... Par conséquent le fait historique n'est pas plus donné que les autres; c'est l'historien, ou l'agent du devenir historique, qui le constitue par abstraction, et comme sous la menace d'une régression à l'infini (1962b, repris in 2008, p. 834-835).

Il est indéniable que l'analyse structurale nous livre les traces d'un passé qui ne disparaît jamais totalement, sans pour autant que l'on en connaisse les causes. Par ailleurs, «le bricolage» qui s'opère au cours du récit d'un mythe détourne parfois les éléments de leur sens et place originels pour leur attribuer une signification qui ne peut s'exprimer que dans le temps présent. De plus, la démarche des ethnologues et des historiens ne peut se faire sans le présupposé que les hommes partagent à travers l'espace ou le temps des propriétés symboliques communes : 
car comment [les historiens] pourraient-ils reconstituer ce qui se passait dans la tête des gens qui vivaient il ya deux ou trois ou quatre siècles s'ils ne commençaient par postuler qu'il y a quelque chose de commun entre eux et nous, et que les hommes pensent fondamentalement de la même façon? Sinon, le passé et le lointain deviendraient pareillement hors d'atteinte (Éribon 1988, p. 174).

Enfin, le comparatisme systémique qui transcende à la fois les objets et les cultures auxquelles ils appartiennent, conduit Lévi-Strauss à des tentatives homologiques qui interpellent. Or il parvient ainsi à démontrer les correspondances logiques qui existent entre les différents pans d'une culture - même si le système comprend nécessairement des disfonctionnements et des contournements de la norme. En présupposant que des sociétés différentes peuvent partager des capacités opératoires communes proprement humaines, il reconnaît ce système logique au-delà des frontières culturelles.

Grâce à la grille structurale offrant l'image d'un monde doté de cohérence, il parvient à donner une réponse scientifique aux questions métaphysiques qui se posaient à lui lorsque, observant la nature et les hommes, il recherchait le sens des objets naturels, des classifications et des institutions humaines, en refusant la seule explication du hasard et de la coïncidence. Son refus d'enfermer l'autre dans sa différence l'a conduit à considérer que la mise au jour de la logique opératoire de schèmes inconscients lui permettrait de comprendre ce qu’un esprit différent avait généré.

Chaque page de l'œuvre de Lévi-Strauss témoigne d'une pensée rigoureuse et de thèmes récurrents qu'il développe d'ouvrages en ouvrages. Il a révélé à notre attention des communautés dont les compétences et les savoir-faire ont définitivement libéré notre propre conception de l'homme de préjugés. Son apport fondamental est d'avoir dépassé l'atomisme et d'avoir considéré les objets dans la relation réciproque qui les unit. À ceux qui veulent connaître l'homme LéviStrauss, il dit avoir donné une clé désormais célèbre dans La potière jalouse (1988, p. 134) où il s'identifie à Don Quichotte «qui a la manie de redresser les torts, de se faire le champion des opprimés et qui éprouve un désir obsédant de retrouver le passé derrière le présent». Il avoue humblement que son rôle a été «de préserver quelque chose de cette richesse et de cette diversité que l'humanité, peut-être connaîtra sous d'autres formes, mais en tous les cas, sous des formes absolument différentes de celles qui ont existé dans le passé » (2000, p. 43). Et il ajoute : «J'ai le sentiment qu'un certain nombre de problèmes ne pourront être traités, mieux que je ne l'ai fait, qu'en passant par ce que j'ai écrit même si c'est pour le démolir. Que mes livres ont marqué un moment de la réflexion ethnologique et qu'on en tiendra compte.» (1988, p. 136).

Son œuvre demeure donc ouverte. Il invite les sciences humaines de demain à prendre en compte les acquis du passé, à rester attentives les unes aux autres, et à développer de nouvelles méthodes pour appréhender et comprendre les manifestations symboliques chez l'humain. Il aura su démontrer que l'homme ne peut être réduit en parcelles de connaissances sans que ces dernières ne se 
rejoignent un jour à l'occasion de quelques problématiques parmi les plus fondamentales de l'anthropologie. Car il ne fait pas de doute pour nous qu'il s'agit de poursuivre sa perspective comparative et pluridisciplinaire.

Pour finir, l'anthropologue nous aura instruits, à travers sa conception de l'homme et au-delà d'une démarche scientifique, d'une sagesse que son œuvre n'aura cessé d'incarner.

\section{RÉFÉRENCES}

Arom, Simha, Nathalie Fernando, Susanne Fürniss, Sylvie LeBomin, Fabrice Marandola et Jean Molino. 2008. «La catégorisation des patrimoines musicaux dans les sociétés de tradition orale». Catégories et catégorisation. Une perspective interdisciplinaire, sous la dir. de Frank Alvarez-Péreyre, SELAF, 448, numéro spécial, 272-313.

Arom, Simha, Nathalie Fernando, et Fabrice Marandola. 2007. «An Innovative Method for the Study of African Scales ». Actes de colloque : 4th Sound and Music Computing Conference, Lefkada, Greece, 11-13 juillet 2007, 107-116.

Barthes, Roland. 1985. Éléments de sémiologie. Paris : Seuil.

Bellour, Raymond et Catherine Clément. 1979. Claude Lévi-Strauss, textes de et sur Claude Lévi-Strauss. Paris : Gallimard.

Cazier, Jean-Philippe (éd.). 2007. ABéCédaire de Claude Lévi-Strauss. Mons : Sils Maria.

Chiarucci, Henri. 1973. «Essai d'analyse structurale d'œuvres musicales», Musique en Jeu, 12 : 11-44.

Donin, Nicolas et Frédéric Keck. 2006. «Lévi-Strauss et la musique. Dissonances dans le structuralisme». Revue d'histoire des sciences humaines, 14 : 101-136.

Éribon, Didier et Claude Lévi-Strauss. 1988. De près et de loin. Paris : Odile Jacob.

Fernando, Nathalie. 2007. «De la dynamique du schème dans les musiques de tradition orale. Problématique de la polymorphie des formes envisagée à travers des répertoires d'Afrique centrale». Musique et schème : entre percept et concept, sous la dir. de Béatrice Ramaut-Chevassus. SaintÉtienne : Publications de l'Université de Saint-Étienne, CIEREC Travaux 131, 239-278.

- - Study of African Scales : A New experimental approach for cognitive aspects». 2006. Approaches to African Musics, sous la dir. de Enrique Càmara Landa et Silvia Martínez García (éd.), Universidad de Valladolid, 57-72. Également publié électroniquement dans Trans en 2007.

-2006. «Expérimenter en ethnomusicologie», L’Homme, numéro spécial 'Musique et anthropologie', sous la dir. de Bernard Lortat-Jacob et Myriam Roving-Olsen, $\mathrm{n}^{\mathrm{O}}$ 171-172 : 285-302.

Géraud, Marie-Odile, Olivier Leservoisier et Richard Pottier. 1998. Les notions clés de l'ethnologie. Paris : Armand Colin/Masson.

Keck, Frédéric. 2005. Claude Lévi-Strauss, une introduction. Paris : La Découverte.

Larc-Lipiansky, Mireille. 1973. Le structuralisme de Lévi-Strauss. Paris : Payot. 
Lévi-Strauss, Claude. 1950. «Introduction à l'œuvre de Marcel Mauss». Marcel Mauss, Sociologie et anthropologie. Paris : Presses Universitaires de France, IX-LII.

-1958. Anthropologie structurale. Paris : Plon.

—1962a. Le totémisme aujourd'hui. Paris : PUF. 1962b. La pensée sauvage. Paris : Plon.

-1964. Mythologiques, Le cru et le cuit. Paris: Plon.

-1967. Mythologiques, Du miel aux cendres. Paris : Plon.

-1967a. Mythologiques, L'homme nu. Paris : Plon.

1968. Mythologiques, L'origine des manières de tables. Paris : Plon.

-1973. Anthropologie structurale II. Paris : Plon.

-1985. La potière jalouse. Paris : Plon.

1993. Regarder, écouter, lire. Paris : Plon.

—. 2008. CEuvres, sous la dir. de Vincent Debaene, Frédéric Keck, Marie Mauzé et Martin Ruell. Paris :Gallimard.

Maranda, Pierre. 1980. «The Dialectic of Metaphor: An Anthropological Essay on Hermeneutics». The Reader in the Text, sous la dir. de Rubin Suleiman et Inge Crosman, 183-204. Princeton : Princeton University Press.

-1993. «Imagination Structuring Processes». The Expert Sign : Semiotics of Culture, sous la dir. de Jan L. Slikkerveer et al., 169-186. Leiden : DSWO Press.

Maranda, Pierre (éd.). 2001. The Double Twist : From Ethnography to Morphodynamics. Toronto : University of Toronto Press.

Massenzio, Marcello. 2002. Entretien, Claude Lévi-Strauss un itinéraire, 26 juin 200o, Paris, L'Échoppe.

Nattiez, Jean-Jacques, «Rencontre avec Lévi-Strauss : le plaisir et la structure», Musique en Jeu, 12 : 3-10.

Piaget, Jean. 1968. Le structuralisme. Paris : PUF.

Rivest, Martin. 2001. De l'éthnologie comme possible humanisme? Autour de Claude Lévi-Strauss et du structuralisme, mémoire de maîtrise. Montréal : Université de Montréal.

Scubla, Lucien. 1998. Lire Lévi-Strauss. Paris : Odile Jacob.

Simonis, Yvan. 1980. Claude Lévi-Srauss ou «la passion de l'inceste». Paris : Flammarion.

Vielle, Christophe, Pierre Swinggers et Guy Jucquois. 1994. Comparatisme, mythologie, langages, en hommage à Claude Lévi-Strauss. Louvain-la Neuve : Peeters.

Filmographie

Beuchot, Pierre. 2006. Lévi-Strauss, Entretiens avec Jean-José Marchand (1972), DVD + DVDRom, Éditions Montparnasse. 


\title{
RÉSUMÉ
}

Le présent article est issu d'une communication orale donnée à l'occasion d'une journée d'étude fêtant le centenaire de Lévi-Strauss. Cette dernière avait pour objectif de synthétiser brièvement la nature de son regard structural et les préoccupations d'ordre anthropologique qui sous-tendent sa réflexion. Elle tentait aussi de retenir les points forts de la recherche lévi-straussienne qui permettent encore aujourd'hui à nombre de sciences humaines de poser de nouveaux enjeux et de les traiter en retenant les leçons de l'histoire.

Face à une œuvre colossale qui a maintes fois été analysée, ma démarche consiste à m'intéresser davantage à ce que Lévi-Strauss révèle de son parcours intellectuel dans son ouvre, mais aussi au cours des multiples entretiens qu'il a donnés - notamment ses sources d'inspiration, ce qu'il dit de ce qu'il a écrit, et la façon dont cela a été reçu, à savoir les critiques qui lui ont été faites mais aussi les réponses qu'il a apportées.

Il s'agit en quelque sorte d'envisager l'homme dans sa démarche d'anthropologue, mais aussi d'évoquer Lévi-Strauss vu par Lévi-Strauss au cœur des sciences humaines, milieu propice - et c'est naturel - aux débats effervescents.

\begin{abstract}
This article is based on a lecture given on the occasion of a workshop celebrating the centenary of Lévi-Strauss. The latter was intended to briefly summarize the nature of his structuralist gaze and the anthropological concerns that underlie his thinking. It also recalled the highlights of Lévi-Strauss's research, which still allow many of the humanities to pose new challenges and face them while retaining the lessons of history.

Faced with a colossal body of work that has been repeatedly analyzed, my approach is to focus on what Lévi-Strauss reveals of his intellectual journey in his work, but also during the many interviews he gave: notably, his sources of inspiration, his opinion of his own writings and how these were received, namely, the criticisms that were made but also the responses he provided.

In a way, it is an attempt to consider the man in his approach as an anthropologist, and also to evoke Lévi-Strauss on Lévi-Strauss at the heart of the humanities, an environment naturally conducive to lively debate.
\end{abstract}

